Bond University

Research Repository

\title{
Presence or Absence of Skeletal Muscle Dysfunction in Chronic Obstructive Pulmonary Disease is Associated With Distinct Phenotypes
}

Cruthirds, Clayton L; Van der Meij, Barbara S; Wierzchowska-McNew, Agata; Deutz, Nicolaas E P; Engelen, Marielle P.

Published in:

Archivos de Bronconeumologia

DOI:

10.1016/j.arbres.2019.12.034

Licence:

CC BY-NC-ND

Link to output in Bond University research repository.

Recommended citation(APA):

Cruthirds, C. L., Van der Meij, B. S., Wierzchowska-McNew, A., Deutz, N. E. P., \& Engelen, M. P. (2021).

Presence or Absence of Skeletal Muscle Dysfunction in Chronic Obstructive Pulmonary Disease is Associated With Distinct Phenotypes. Archivos de Bronconeumologia, 57(4), 264-272.

https://doi.org/10.1016/j.arbres.2019.12.034

\section{General rights}

Copyright and moral rights for the publications made accessible in the public portal are retained by the authors and/or other copyright owners and it is a condition of accessing publications that users recognise and abide by the legal requirements associated with these rights.

For more information, or if you believe that this document breaches copyright, please contact the Bond University research repository coordinator 


\section{ABSTRACT}

Introduction: Reduced skeletal muscle function and cognitive performance are common extrapulmonary features in Chronic Obstructive Pulmonary Disease (COPD) but their connection remains unclear. Whether presence or absence of skeletal muscle dysfunction in COPD patients is linked to a specific phenotype consisting of reduced cognitive performance, comorbidities and nutritional and metabolic disturbances needs further investigation.

Methods: Thirty-seven patients with COPD (grade II-IV) were divided into two phenotypic cohorts based on the presence (COPD dysfunctional, $n=25$ ) or absence (COPD functional, $n=12$ ) of muscle dysfunction. These cohorts were compared to 28 healthy, age matched controls. Muscle strength (dynamometry), cognitive performance (Trail Making Test and STROOP Test), body composition (Dual-energy X-Ray Absorptiometry), habitual physical activity, comorbidities and mood status (questionnaires) were measured. Pulse administration of stable amino acid tracers was performed to measure whole body production rates.

Results: Presence of muscle dysfunction in COPD was independent of muscle mass or severity of airflow obstruction but associated with impaired STROOP Test performance $(p=0.04)$, reduced resting $\mathrm{O}_{2}$ saturation $(p=0.003)$ and physical inactivity $(p=0.01)$, and specific amino acid metabolic disturbances (enhanced leucine $(p=0.02)$ and arginine $(p=0.06)$ production). In contrast, COPD patients with normal muscle function presented with anxiety, increased fat mass, plasma glucose concentration, and metabolic syndrome related comorbidities (hypertension and dyslipidemia). 
Conclusion: COPD patients with muscle dysfunction show characteristics of a cognitive - metabolic impairment phenotype, influenced by the presence of hypoxia, whereas those with normal muscle function present a phenotype of metabolic syndrome and mood disturbances. 
Key Words: COPD, muscle strength, cognition, phenotype, metabolism

\section{Abbreviations}

BMI: Body Mass Index

DXA: Dual-energy X-ray Absorptiometry

FM: Fat Mass

FFM: Fat Free Mass

SCWT: Stroop Color Word Test

TMT: Trail Making Test

MPE: Maximal expiratory pressure

MIP: Maximal inspiratory pressure

HADS: Hospital Anxiety and Depression Scale

PASE: Physical Activity Scale for the Elderly

BCAA: Branched-Chain Amino Acids

BCKA: Branched-Chain Keto Acids

WBP: Whole Body Production 


\section{INTRODUCTION}

Skeletal muscle dysfunction is an extrapulmonary feature of Chronic Obstructive Pulmonary Disease (COPD)\{Jaitovich, 2018, 175-186;Gea, 2015, E418-38\}, negatively affecting their physical activity, quality of life, and mortality\{Maltais, 2014, e15-62\}. Muscle dysfunction in COPD can be present independent of lung function severity\{Swallow, 2007, 115-20\} and muscle mass loss, indicative of muscle myopathy. Moreover, cognitive impairment\{Schure, 2016, 46-52\} and mood disturbances\{Ouellette, $2017,639-650\}$ are present in COPD at higher rates than in the general (non-COPD) population\{Divo, 2015, 640-50\}. A link between the muscle and brain has been observed in older adults as reflected by the simultaneous decline in both muscle strength and cognitive function\{Raji, 2005, 1462-8;Alfaro-Acha, 2006, 859-65\}, and the fact that exercise training can improve cognitive function\{Pereira, 2011, 279-85;Teixeira, $2013,148-156\}$. Presence of physical inactivity and systemic inflammation in COPD are known risk factors for both physical and cognitive dysfunction\{Barreiro, 2015, 41326;Plassman, 2010, 182-93;Degens, 2010, 28-38;Baierle, 2015, 1-12\}. In addition, hypoxia may contribute to the high prevalence of muscle dysfunction and impaired cognitive performance in COPD, as hypoxia is known to increase oxidative stress and inflammation, leading to signaling remodeling of smooth muscle in COPD\{CoimbraCosta, 2017, 216-225;Wiegman, 2015, 769-80\} and physical damage of brain tissue\{Ryu, 2013, 357-66\}. In line, COPD patients on long-term oxygen therapy exhibited more cognitive impairment than non-oxygen users despite similar disease severity\{Karamanli, 2015, 2087-94\}. These physiological changes suggest a connection 
between muscle and cognitive dysfunction in patients with COPD but whether there is a direct link remains unclear.

Both fibre type shifts towards a predominantly type II composition and disturbances in protein and amino acid metabolism (e.g. glutamate and the branched chain amino acids)\{Engelen, 2001, 859-64\} in COPD may contribute to the reduced functional metabolic capacity of the limbs\{Ciciliot, 2013, 2191-2199\} in these patients. Whether disturbances in the metabolism of these as well as other amino acids (including tryptophan as precursor of serotonin)\{Lieben, 2018, 321-328\}) provide a direct metabolic link between the presence of muscle and cognitive dysfunction in COPD as recently suggested\{Gosker, 2019,915$\}$ deserves further investigation. We hypothesize that particularly COPD patients with muscle dysfunction are at risk for cognitive impairment due to the presence of disease related risk factors such as hypoxia, inflammation, and physical inactivity contributing to specific disturbances in amino acid metabolism.

In the present study, we examined a heterogeneous group of moderate to severe COPD patients who were stratified based on the presence/absence of muscle dysfunction into dysfunctional and functional COPD cohorts. Cognitive performance, mood status, disease severity, habitual dietary intake and physical activity, comorbidities, protein and amino acid metabolism, and body composition were examined in both groups and compared to a group of healthy age-matched subjects from the MEDIT (MEtabolism of Disease with Isotope Tracers) trial, to examine whether presence or absence of skeletal muscle dysfunction in COPD patients is linked to a specific phenotype. 


\section{MATERIALS AND METHODS}

\section{Subjects}

We studied 37 older adults with a clinical diagnosis of moderate to severe airflow obstruction (grade II-IV), according to the established Global Initiative for Chronic Obstructive Pulmonary Disease GOLD guidelines and 28 healthy age matched subjects. The participants were recruited via advertisements in the surrounding hospitals and local community. Patients were clinically stable and not suffering from an acute exacerbation or infection and their disease was stable for the previous four weeks. Exclusion criteria were pre-existent untreated metabolic or renal disease, malignancy, recent surgery, and use of systemic corticosteroids one month prior to the study. Medical history and medication use were assessed as part of the screening process. Written informed consent was obtained from all subjects, and the study was approved by the Institutional Review Board of Texas A\&M University.

\section{Anthropometrics, body composition, and lung function}

Body weight and height were measured by a digital beam scale and stadiometer, respectively, and regional values for fat mass and fat-free mass were obtained from all subjects while in supine position, by Dual-Energy X-ray Absorptiometry (DXA) (Hologic QDR 4500/ Version 12.7.3.1 (Bedford, MA)). Anthropometric and body composition measures were standardized for height $(\mathrm{kg} / \mathrm{m} 2)$, to obtain BMI, FFM index (FFMI), FM index (FMI), and appendicular skeletal muscle index (ASMI). Post-bronchodilator forced 
expiratory volume in 1 second $\left(F E V_{1}\right)$ was assessed with the highest value from $\geq 3$

technically acceptable maneuvers.

\section{Muscle function testing}

The single leg muscle function test was completed with the right limb, using an isokinetic dynamometer (Isokinetic International, Chattanooga, TN). In a seated position the trunk, pelvis, and right thigh were secured using straps to prevent body movement. Standardized, intense verbal encouragement was given to all subjects throughout the test. All tests were performed by the same researcher to secure consistency. After a warm-up (10 low-effort repetitions), peak leg torque was assessed at $60^{\circ} / \mathrm{sec}$ by 5 maximal extension-flexion cycles, each cycle followed by ten seconds of rest.

Handgrip strength was assessed by Vernier Hand Dynamometry (Vernier Software and Technology, Beaverton, OR) with subjects in a seated position with their dominant arm unsupported and elbow flexed at 90 degrees. Subjects were encouraged to squeeze the dynamometer maximally for 5 seconds with 60 seconds rest between each trial to obtain at least 3 reliable measurements.

Maximal expiratory pressure (MEP) and inspiratory pressure (MIP) as measures of respiratory muscle strength were assessed by determining the maximal value of at least 3 reliable attempts using a hand-held mouth pressure device (Micro Respiratory Pressure Meter (RPM)) with at least 1 minute of rest between each attempt.

\section{Cognitive testing and questionnaires}


Trail Making Test (TMT) was used to assess visual-motor tracking skills and psychomotor speed. The subjects had to connect consecutive numbers randomly arranged on a page (TMT- Pt A) or consecutive numbers and letters in alternating order (TMT- Pt B).

Stroop Color Word Test (SCWT) was used to measure executive functioning and cognitive flexibility as response inhibition for colored printed words. The completion times (sec) and number of errors were recorded for each part. Mood status (depression and anxiety) was assessed by the Hospital Anxiety and Depression Scale (HADS).

Habitual dietary intake was assessed using 24-hour dietary recall while daily physical activity level was measured by the Physical Activity Scale for the Elderly questionnaire (PASE). The Charlson index was used for the assessment of concomitant comorbidities.

\section{Stable isotope administration by IV pulse}

A peripheral line was placed in a superficial dorsal vein of the hand or lower arm for infusion of a bolus of stable tracers and subsequent blood sampling. The hand was placed in a thermostatically controlled hot box (internal temperature: $60^{\circ} \mathrm{C}$ ), a technique to mimic direct arterial sampling. After a venous blood sample was collected to measure baseline enrichment, an intravenous pulse administration of a cocktail of stable tracers\{Deutz, 2018, 167-178\} was given (Figure 1) (Cambridge Isotopic Laboratories, Woburn, MA, USA). Arterialized-venous blood was sampled at multiple time points until four hours after pulse administration.

\section{Biochemical analysis and calculations}


Arterialized-venous blood was put in Li-heparinized (Becton Dickinson Vacutainer system, Franklin Lakes, New Jersey, USA), immediately put on ice to minimize enzymatic reactions, and centrifuged to obtain plasma. A part of the plasma was aliquoted into tubes with trichloroacetic acid for denaturation of proteins. Samples were immediately frozen and stored at $-80^{\circ} \mathrm{C}$ until further analysis. Tracer enrichments [tracer:tracee ratio (TTR)] and plasma amino acid concentrations were analyzed batch-

wise by LC-MS/MS or GC-MS/MS as previously reported\{Jonker, 2016, 1458-64;Deutz, $2018,167-178\}$. Whole body production (WBP) rates of various amino acids (e.g.leucine, tryptophan, glutamate, glutamine, glycine, arginine, citrulline) were calculated from the intravenously administered pulse as done previously\{Deutz, 2018, 167-178\}.

\section{Statistical analysis}

We determined gender specific normal values for single leg and handgrip strength based on the measurements obtained from $38(18 \mathrm{~F} / 20 \mathrm{M})$ healthy older subjects who visited CTRAL in the past 3 years. These values were used to create two COPD cohorts: COPD muscle functional (> 1SD below normal value for handgrip and/or leg strength, $n=12$ ) and COPD dysfunctional ( $<$ SD below normal value, $n=25)$. Results are expressed as mean \pm standard error (SE). The unpaired Student's $t$ test was used to determine differences between healthy older adults and the total COPD group. Oneway Analysis of variance (ANOVA), followed by Bonferroni multiple comparison test, was used to determine differences between healthy older adults, COPD muscle functional, and COPD dysfunctional cohorts. Pearson's correlation analysis was used to 
determine relationships between muscle function and disease severity, cognitive performance, mood status, habitual dietary intake and physical activity, comorbidities, protein and amino acid metabolism, and body composition variables. The statistical package within Graphpad Prism (Version 8.2.0, GraphPad Software Inc, San Diego, USA) was used for data analysis. The level of significance was set a priori at $p<0.05$.

\section{RESULTS}

\section{Body composition, disease severity, and habitual dietary intake}

General subject characteristics are presented in Table 1. The muscle dysfunction COPD cohort was older than the functional and tended to be older $(p=0.055)$ than the healthy group. There was a slight gender skew in the functional cohort with $75 \%$ being female as compared to 46 and $48 \%$ in healthy and dysfunctional, respectively. BMI tended to be higher in the functional COPD group than in the other two groups but no differences were found in lean mass (Figure 2a). The functional COPD group tended to have more total fat mass compared to the healthy $(p=0.058)$ with increased percentage android and gynoid fat mass over the dysfunctional group. Charlson comorbidity index was elevated in both COPD cohorts. No differences in severity of airflow obstruction were seen between COPD cohorts (Figure $\mathbf{2 b}$ ) except for a trend towards a lower resting oxygen saturation in the dysfunctional group. Number of oxygen users (defined as $\mathrm{O}_{2}$ use continuously, at night or as needed) was $52 \%$ and $33 \%$ in the dysfunctional and functional COPD group, respectively. Daily dietary caloric and macronutrient intake was similar across all groups for all nutrients measured except for lower fiber intake in both COPD cohorts. 


\section{Muscle function and physical activity}

Maximal handgrip and leg strength were lower in the COPD total group (Table 2). In-line with group allocation, leg strength was lower in the COPD dysfunctional group than in the healthy and functional COPD cohort, but no difference was found between the healthy and functional COPD groups. Leg strength corrected for total lean mass, as a measure of muscle quality, was $30 \%$ lower in the COPD dysfunctional group (Table 2). Inspiratory and expiratory muscle strength along with daily physical activity were lower in COPD total as compared to healthy, due to lower values in the dysfunctional COPD cohort.

\section{Cognitive performance and mood status}

SCWT assessed executive functioning and cognitive flexibility while TMT evaluated visual-motor tracking skills and psychomotor speed (Table 2, Figure 3). The total COPD group performed significantly slower on all three parts of the SCWT in addition to the TMT parts, however after stratification, significance only remained for the dysfunctional COPD cohort, with a trend towards a difference between functional and dysfunctional for TMT-Pt A. The functional and dysfunctional COPD cohorts reported to be more depressed than the healthy group with the functional COPD cohort being most anxious.

\section{Protein and amino acid metabolism}

No differences were found in plasma amino acid concentrations among the healthy and COPD groups (Table 3), although both COPD cohorts had elevated plasma hs-CRP levels, whereas plasma glucose levels were increased only in the functional COPD 
cohort. Whole body production of the Branched-Chain Amino Acid (BCAA) leucine was elevated in the dysfunctional cohort compared to healthy while the amino acids citrulline and arginine tended to be increased $(p=0.02, p=0.05, p=0.06$, respectively, Table 4). No differences were observed in the production rates of the remaining amino acids.

\section{$\underline{\text { Relationships between muscle function, cognitive performance, and metabolic }}$}

\section{markers in COPD}

\section{Muscle function}

Reduced single leg strength per kilogram lean mass in COPD was associated with cognitive dysfunction as displayed by increased time to completion for SCWT-Pt 3

(Figure 5a) and TMT-Pt B (Figure 5b) and lower plasma tryptophan concentration (Figure 4d). Higher single leg strength alone was associated with a higher plasma glutamate concentration $(r: 0.47, p<0.01)$ and lower values for glycine whole body production $(r:-0.40, p<0.05)$ and plasma concentration $(r:-0.40, p<0.05)$.

\section{Cognitive performance}

Increased time to completion for SCWT-Pt3 was associated with metabolic disturbances as reflected by lower values for total plasma concentration of Branched-Chain Keto Acids (BCKAsum) $(r:-0.43, p<0.05)$. Likewise, TMT-Pt B was associated with lower values for plasma concentrations of LEU ( $r:-0.48, p<0.01)$, VAL $(r:-0.40, p<0.05)$, BCAAsum (Figure 4c), and BCKAsum (r:-0.38, p<0.05).

\section{DISCUSSION}


We studied whether COPD patients are characterized by distinct phenotypes based on the presence/absence of muscle dysfunction relative to healthy, age-matched controls. We found that COPD patients with muscle dysfunction are characterized by decreased cognitive performance, mood status, physical activity, and transcutaneous $\mathrm{O}_{2}$ saturation. COPD patients with preserved muscle function presented characteristics of metabolic syndrome and mood disturbances. Presence of muscle dysfunction in COPD was independent of muscle mass loss and severity of airflow obstruction.

\section{Profile of COPD patients with muscle dysfunction phenotype}

Our muscle dysfunction COPD cohort displayed systemic weakness as reflected by concurrent weakness of the upper, lower, and respiratory muscles but no differences were seen in muscle mass across the study groups. Muscle weakness despite preserved muscle mass has been seen in COPD patients previously and supports a more rapid development of muscle myopathy before atrophy develops\{Jaitovich, 2018, 175-186\}. Muscle quality, i.e. strength relative to size, was $>30 \%$ lower in our dysfunctional cohort as compared to controls. As muscle mass can not be used as a proxy of muscle function in COPD and muscle dysfunction is a predictor of morbidity and mortality in these patients, incorporation of muscle function testing to identify muscle weakness is therefore of critical importance. A recent review highlighted the combination of etiological factors (i.e. inactivity, comorbidities, inflammation) and biological mechanisms (i.e. oxidative stress, proteolysis) which promote dysfunction of ventilatory and peripheral muscles in COPD\{Barreiro, 2016, 297-311\}. A holistic approach to prevent and reverse muscle dysfunction in COPD is therefore 
required\{Jaitovich, 2018, 175-186\}, in which the treatment strategy focuses on employing resistance and endurance exercise in combination with optimized diet and medications.

\section{- Reduced cognitive function}

Patients with muscle dysfunction showed reduced time to completion and increased number of errors across both the SCWT and TMT, which are reflective of impaired executive functioning with cognitive inflexibility and reduced psychomotor speed, respectively. Decreased productivity on processing-speed tasks such as the SCWT is an important indicator of bradyphrenia characterized by reduced processing speed, inattentiveness, delayed response and psychomotor impairment. Disturbances in mood state were also seen in our muscle dysfunctional cohort in agreement with previous studies reporting the severity of anxiety and depression increases with the appearance of COPD symptoms and reduction in daily physical activity\{Miravitlles, 2014, 259-65\}. Previous studies showed that $18-35 \%$ of COPD patients present with cognitive impairment\{Ouellette, 2017, 639-650\}. Patients with concomitant muscle and cognitive dysfunction are in danger of being overlooked as cognitive impairment is a potential exclusion criterion for pulmonary rehabilitation\{Cleutjens, 2017, 420-426\}.

In a longevity-based study, a strong association was observed between a reduction in cognitive function and handgrip strength in older adults, with the lowest cognitive performers having the steepest decline in handgrip strength\{Raji, 2005, 14628\}. The authors proposed inflammation as a shared factor affecting both cognition and muscle function. In a follow up study using HEPESE data, the same trend was seen over a 7 year follow up\{Alfaro-Acha, 2006, 859-65\}. The brain connects cognitive and 
muscle function via the central nervous system so a decline in performance of both activities may reflect reduced integrity of the nervous system. Shared pathogenic factors between cognitive and muscle function e.g. oxidative stress, inflammatory markers, and sex steroids further link these functional parameters\{Rosano, 2005, 8-14\}.

Interestingly, older adults completing 16 weeks of exercise training improved cognitive function independent of sufficient cardiovascular stimulus to affect aerobic capacity or specific cognitive training\{Teixeira, 2013, 148-156\}. Enhanced cerebral blood flow, synthesis of neurotransmitters, or regulation of neurotrophic factors are possible causes of this concomitant improvement. As 6-min walk distance is positively associated with cognitive function in COPD\{Dag, 2016, 1044-50\}, our data support the inclusion of cognitive assessments in the systemic evaluation of exercise rehabilitation strategies.

Disease related factors (systemic inflammation and hypoxemia) to explain the link between muscle and cognitive dysfunction in COPD

Although lung function parameters were comparable between COPD cohorts, resting oxygen saturation tended to be lower in COPD patients with muscle dysfunction. Additionally, nearly $40 \%$ of the muscle dysfunctional patients were oxygen users vs. $16 \%$ of functional. Significant associations were found in our total COPD population between oxygen saturation and both cognitive performance and muscle quality, confirming previous findings that hypoxia is an important systemic factor underlying whole body functional performance in COPD $\{K i m, 2008,637-58\}$. Likewise, low oxygen saturation, but not disease severity (e.g. FEV1, BODE), puts COPD patients at an 
increased risk for muscle dysfunction and cognitive impairment\{Thakur, 2010, 263-9\}, as hypoxia increases free radical production, leading to physical damage of both muscle\{Takabatake, 2000, 1179-1184\} and brain tissue\{Ryu, 2013, 357-66\}.

Furthermore, higher plasma hs-CRP concentrations were seen in the dysfunctional cohort indicative of an increased systemic inflammatory state. Inflammation is one of the highest-ranking risk factors for muscle dysfunction\{Degens, 2010, 28-38\} and cognitive impairment\{Baierle, 2015, 1-12\}. Even slightly increased levels of inflammatory markers correlated with reduced cognitive performance in healthy older adults, metabolic syndrome and Alzheimer's disease\{Pelgrim, 2019, 99-110\}. In skeletal muscle, TNFa, a proinflammatory cytokine, can activate the NF-KB pathway thereby upregulating the expression of inducible nitric oxide synthase which facilitates the degradation of myosin heavy chains through the ubiquitin-proteasome complex\{Langen, 2004, 227-237\}.

- Alterations in amino acid metabolism to explain muscle and cognitive dysfunction in COPD

While weight status was not a selection criteria, virtually all our COPD patients displayed no markers of cachexia (e.g. reduced BMI or muscle mass) and had an adequate daily protein intake ( $>0.8 \mathrm{~g} / \mathrm{kg} /$ day). Nevertheless, metabolic alterations of amino acids that play a role in muscle contractility were seen. Increased whole body production of arginine (precursor of nitric oxide synthesis) and its precursor citrulline were observed in the dysfunctional COPD group, in agreement with our previous data in COPD JJonker, 2016, 1458-64\}. Reduced muscle function was also associated with increased production of glycine (precursor of creatine and glutathione and modulator of protein synthesis), and increased plasma concentration of glutamate (neurotransmitter, 
and role in tricarboxylic acid and purine nucleotide cycle). In line with our previous findings\{Engelen, 2012, 616-624\}, we observed an increased whole body production of leucine in our muscle dysfunctional cohort. Leucine plays an important role in muscle protein degradation and synthesis, and BCAA supplementation in COPD resulted in improved cognitive function\{Dal Negro, 2012, 67-75\}. In line, the impaired cognitive performance in our COPD patients with muscle dysfunction was associated with lower plasma concentrations of BCAA and BCKA, suggesting a BCAA metabolic link between cognitive and muscle dysfunction. Cognitive dysfunction has been associated with abnormalities in metabolism of the neuroactive amino acid tryptophan\{Lieben, 2018, 321-328\} (precursor of serotonin) in multiple other conditions. Although no significant alterations were seen in tryptophan production in our dysfunctional COPD cohort group, an association was found between reduced TRP concentrations and muscle strength. In line, impaired kynurenine (tryptophan metabolite) metabolism has recently been seen in skeletal muscle of COPD patients\{Gosker, 2019, 915\}, and kynurenine disturbances have been associated with impaired cognition and depression\{Solvang, 2019, 155-162\}. Whether there is a direct metabolic link between muscle and cognitive dysfunction via alterations in the BCAA and TRP pathways needs further investigation in COPD.

\section{Profile of COPD patients with normal muscle function}

Patients with normal muscle function had higher BMI due to increased fat mass in both the android and gynoid region. Besides increased android fat mass, higher prevalence of hypertension and dyslipidemia and increased plasma glucose were found in this group, suggesting (early) signs of metabolic syndrome. Metabolic syndrome is of clinical importance in COPD as it has a prevalence of $>30 \%$ in the COPD community with 
higher rates in females\{Cebron Lipovec, 2016, 399-406\}. Our muscle functional COPD cohort presented a phenotype similar to the previously described 'Metabolic'

cluster'\{Vanfleteren, 2013, 728-35\}, characterized by high prevalence of obesity, hypertension, hyperglycemia, and dyslipidemia with comparable lung function. Interestingly, no metabolic alterations were found in this cohort.

\section{Conclusion: Future/past of COPD phenotyping}

In the past decade, approaches to cluster or phenotype COPD subjects have used a wide range of methodologies from detailed clinical history to mathematical based algorithms\{Burgel, 2017, 1701034; Vanfleteren, 2013, 728-35\}. Previously these analyses have been based on clinically relevant outcomes which are easily obtained in a hospital setting, enhancing the potential implementation of these phenotypes for treatment, however muscle functional capacity was not always considered. Our data show the importance of examining muscle and cognitive function in addition to medical history and disease severity, and that two distinct phenotypes exist in COPD based on the presence of absence of muscle dysfunction which needs to be considered when examining and treating patients with COPD.

\section{Acknowledgements}

We sincerely thank all the subjects for their willingness to participate in this research study and all CTRAL personnel who have made this work possible.

Conflicts of Interest: The authors declare no conflicts of interest. 
Source of Funding: 


\section{Legends}

Figure 1. Overview of study design. Cognitive testing: executive functioning and psychomotor speed. Questionnaires: physical activity, mood status, dietary recall. Muscle dynamometry: upper and lower limb muscle strength.

Figure 2. Group characteristics in healthy controls, functional COPD patients, and dysfunctional COPD patients. (A) Appendicular skeletal muscle index. (B) Forced expiratory volume in 1 second. (C) Leg strength per total kilogram lean body mass. Mean \pm SE, statistics were obtained by using 1 -factor ANOVA to compare differences between groups. ${ }^{* * * *} \mathrm{P}<0.0001, \# \# \mathrm{P}<0.001$.

Figure 3. Cognitive function in healthy controls, functional COPD patients, and dysfunctional COPD patients. (A) Time to complete SCWT part 3. (B) Errors during SCWT part 3. (C) Time to complete TMT part A. (D) Time to complete TMT part B Mean $\pm \mathrm{SE}$, statistics were obtained by using 1-factor ANOVA to compare differences between groups. ${ }^{* *} \mathrm{P}<0.01,{ }^{* *} \mathrm{P}<0.05, \# \mathrm{P}<0.05$.

Figure 4. Correlations between $(A, B)$ oxygen saturation and $(C, D)$ amino acid metabolism and functional capacity in all COPD patients. Statistics by Pearson correlation.

Figure 5. Correlations between muscle function (Leg strength/Kg lean mass) and (A) Stroop Color Word Test performance (SCWT - Pt 3). (B) Trail making test performance (TMT- Pt B) in all COPD patients. Statistics by Pearson correlation. 


\begin{tabular}{|c|c|c|c|c|}
\hline & Healthy $(n=28)$ & $\begin{array}{l}\text { COPD total } \\
(n=37)\end{array}$ & $\begin{array}{l}\text { COPD functional } \\
\qquad(n=12)\end{array}$ & $\begin{array}{c}\text { COPD } \\
\text { dysfunctional } \\
(n=25)\end{array}$ \\
\hline Age (years) & $66.6(1.4)$ & $68.4(1.7)$ & $61.8(2.4)$ & $\begin{array}{c}71.6(2.0) \\
* p=0.055 ; \#\end{array}$ \\
\hline Gender (n, female/male) & $13 / 15$ & $20 / 17$ & $8 / 4$ & $12 / 13$ \\
\hline Body Mass Index $\left(\mathrm{kg} / \mathrm{m}^{2}\right)$ & $27.8(0.7)$ & $29.3(1.2)$ & $\begin{array}{l}32.2(2.4) \\
*_{p}=0.058\end{array}$ & $\begin{array}{l}27.9(1.4) \\
\# p=0.067\end{array}$ \\
\hline Fat Free Mass Index $\left(\mathrm{kg} / \mathrm{m}^{2}\right)$ & $18.4(0.6)$ & $18.2(0.5)$ & $18.87(1.0)$ & $17.9(0.6)$ \\
\hline Fat Mass Index $\left(\mathrm{kg} / \mathrm{m}^{2}\right)$ & $8.7(0.5)$ & $10.3(0.8)$ & $12.1(1.6)^{*}$ & $\begin{array}{c}9.4(0.9) \\
\# p=0.072\end{array}$ \\
\hline Android fat (\%) & $35.3(1.5)$ & $36.2(2.2)$ & $41.6(2.8)$ & $\begin{array}{l}33.6(2.8) \\
\# p=0.052\end{array}$ \\
\hline Gynoid fat (\%) & $34.1(1.6)$ & $35.6(1.4)$ & $\begin{array}{l}39.5(1.6) \\
*_{p}=0.067\end{array}$ & 33.6 (1.9)\# \\
\hline $\begin{array}{l}\text { Charlson comorbidity index } \\
\text { (score) }\end{array}$ & $0.38(0.2)$ & $1.61(0.2)^{*}$ & $1.88(0.3)^{*}$ & $1.47(0.2)^{*}$ \\
\hline \multicolumn{5}{|c|}{ Pulmonary function and COPD related measures } \\
\hline $\mathrm{FEV}_{1}$ (\% of predicted) & $98.2(2.5)$ & $44.6(2.6)^{* * *}$ & $45.8(4.6)^{* * *}$ & $44.1(3.2) * * *$ \\
\hline FVC (\% of predicted) & $90.78(2.1)$ & $56.2(2.3)^{* * *}$ & $55.8(3.2)^{* * *}$ & $56.4(3.2) * * *$ \\
\hline $\mathrm{FEV}_{1} / \mathrm{FVC}$ (ratio) & $89.1(2.4)$ & $57.4(2.1)^{*}$ & $61.8(3.8)^{*}$ & $55.3(2.4)^{*}$ \\
\hline Gold Stage (0-4) & $0.00(0.00)$ & $2.8(0.2) * * *$ & $2.8(0.2) * * *$ & $2.8(0.2) * * *$ \\
\hline Oxygen saturation (\%) & $97.7(0.2)$ & $94.2(0.6)^{* * *}$ & $95.4(0.5)^{*}$ & $\begin{array}{c}93.7(0.8)^{* * *} ; \\
\quad \# p=0.103\end{array}$ \\
\hline $\mathrm{O}_{2}$ use $(\mathrm{n}$, yes/no) & $0 / 28$ & $17 / 20$ & $4 / 8$ & $13 / 12$ \\
\hline
\end{tabular}




\begin{tabular}{|c|c|c|c|c|}
\hline \multicolumn{5}{|l|}{ Dietary intake } \\
\hline Total calories (kcal) & 2063 (145) & $1953(106)$ & $2021(193)$ & 1916 (129) \\
\hline Total fat intake (g) & $89.4(10.5)$ & $81.5(7.1)$ & $84.2(11.8)$ & $80.0(9.1)$ \\
\hline Total carbohydrate intake (g) & $217.5(19.0)$ & $213.5(14.5)$ & $220.5(24.0)$ & $209.6(18.5)$ \\
\hline Total fiber intake (g) & $21.8(2.2)$ & $14.6(1.3)^{* *}$ & $14.9(1.8)^{*}$ & $14.5(1.7)^{* *}$ \\
\hline Total protein intake (g) & $87.3(7.0)$ & $\begin{array}{l}73.0(4.9) \\
*_{p}=0.093\end{array}$ & $73.7(8.3)$ & $72.7(6.3)$ \\
\hline Protein intake (grams $/ \mathrm{kg} /$ day) & $1.1(0.7)$ & $0.97(0.08)$ & $0.88(0.10)$ & $1.0(0.1)$ \\
\hline \multicolumn{5}{|c|}{$\begin{array}{l}\text { Data are mean (SE). Statistics are by unpaired t-test or 1-way ANOVA. FEV } 1 \text { : Forced Expiratory Volume in } \\
\text { one second. FVC: Forced Vital Capacity. Android fat and gynoid fat correspond to central and peripheral fat } \\
\text { distribution, respectively. }{ }^{*} \text { denotes difference from Healthy, } p<0.05 ; \text { \# denotes difference from COPD } \\
\text { functional, } p<0.05 \text {. }\end{array}$} \\
\hline
\end{tabular}


Table 2 - Muscle function, cognitive performance and mood status

\begin{tabular}{|c|c|c|c|c|}
\hline & Healthy $(n=28)$ & $\begin{array}{l}\text { COPD total } \\
(n=37)\end{array}$ & $\begin{array}{l}\text { COPD } \\
\text { functional } \\
(n=12)\end{array}$ & $\begin{array}{l}\text { COPD } \\
\text { dysfunctiona } \\
\text { I ( } n=25)\end{array}$ \\
\hline Leg extension torque $(\mathrm{Nm})$ & $104.4(6.1)$ & $72.1(4.4)^{*}$ & $94.2(8.7)$ & $61.5(3.3)^{*} \#$ \\
\hline Leg extension force $(\mathrm{N})$ & $327.6(17.8)$ & $\begin{array}{c}238.1 \\
(14.2)^{* * *}\end{array}$ & $307.2(31.4)$ & 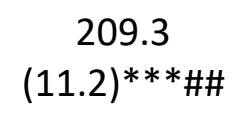 \\
\hline $\begin{array}{l}\text { Leg extension force per } \mathrm{kg} \text { lean mass } \\
(\mathrm{N} / \mathrm{kg})\end{array}$ & $6.5(0.2)$ & $4.9(0.2)^{* * *}$ & $6.1(0.3)$ & $\begin{array}{c}4.5 \\
(0.2)^{* * *} \# \# \#\end{array}$ \\
\hline Hand grip force $(\mathrm{N})$ & $278.4(15.3)$ & $208.7(12.1)^{* *}$ & $\begin{array}{c}225.2(27.6) \\
*_{p}=\mathbf{0 . 0 7 4}\end{array}$ & $\begin{array}{c}202.1 \\
(13.8)^{* *}\end{array}$ \\
\hline Inspiratory muscle strength $\left(\mathrm{cmH}_{2} \mathrm{O}\right)$ & $94.5(7.1)$ & $66.4(4.9)^{* *}$ & $78.5(9.6)$ & $60.9(5.3)^{* * *}$ \\
\hline Expiratory muscle strength $\left(\mathrm{cmH}_{2} \mathrm{O}\right)$ & $113.3(7.7)$ & $92.6(5.2)^{*}$ & $100.1(9.5)$ & $89.5(6.2)^{*}$ \\
\hline PASE (score) & $176.9(18.0)$ & $129.2(10.3)^{*}$ & $141.6(23.9)$ & $122.5(9.4)^{*}$ \\
\hline \multicolumn{5}{|l|}{ Cognition } \\
\hline SCWT: Part 1 time (Sec) & $48.4(1.5)$ & $55.3(2.5)^{*}$ & $52.3(3.5)$ & $56.7(3.3)^{*}$ \\
\hline SCWT: Part 2 time (Sec) & $62.0(2.2)$ & $\begin{array}{l}69.7(2.8) \\
*_{p}=0.051\end{array}$ & $67.1(5.0)$ & $70.9(3.5)^{*}$ \\
\hline SCWT: Part 3 time (Sec) & $110.6(4.6)$ & $134.7(6.8)^{*}$ & $123.1(11.1)$ & $140.1(8.4)^{*}$ \\
\hline SCWT: Interference (Sec) & $55.4(3.7)$ & $72.2(6.0)^{*}$ & $63.4(9.4)$ & $76.3(7.6)^{*}$ \\
\hline SCWT: Part 1 errors & $0.04(0.04)$ & $0.46(0.27)$ & $0.18(0.12)$ & $0.58(0.39)$ \\
\hline SCWT: Part 2 errors & $0.29(0.11)$ & $0.66(0.21)$ & $0.82(0.38)$ & $0.58(0.25)$ \\
\hline
\end{tabular}




\begin{tabular}{|l|c|c|c|c|}
\hline SCWT: Part 3 errors & $0.96(0.35)$ & $4.7(1.2)^{*}$ & $1.8(1.0)$ & $6.0(1.6)^{* * \#}$ \\
\hline TMT: Part A time (Sec) & $27.3(1.7)$ & $35.7(2.5)^{*}$ & $29.9(3.2)$ & $\begin{array}{c}38.4(3.2)^{*} \\
\# p=0.082\end{array}$ \\
\hline TMT: Part B time (Sec) & $58.4(3.8)$ & $80.9(6.3)^{*}$ & $68.0(7.2)$ & $87.0(8.5)^{*}$ \\
\hline HADS: Depression & $2.9(0.7)$ & $5.9(0.6)^{*}$ & $7.0(1.2)^{*}$ & $5.3(0.6)^{*}$ \\
\hline HADS: Anxiety & $4.0(0.7)$ & $\begin{array}{c}5.8(0.6) \\
* p=0.061\end{array}$ & $7.8(1.2)^{*}$ & $4.7(0.6) \#$ \\
\hline
\end{tabular}

Data are mean (SE). Statistics are by unpaired t-test or 1-way ANOVA. SCWT: Stroop Color Word Test. SCWT Interference: (Part 3-((Part 1+Part2)/2). TMT: Trail Making Test. HADS: Hospital Anxiety and Depression Scale. PASE: physical activity questionnaire for the elderly. * denotes difference from Healthy, $p<0.05$; \# denotes difference from COPD functional, $p<0.05$ 


\begin{tabular}{|c|c|c|c|c|}
\hline \multicolumn{4}{|l|}{ Table 3: Plasma concentrations } & \multirow[b]{2}{*}{$\begin{array}{c}\text { COPD } \\
\text { dysfunctional } \\
(n=25)\end{array}$} \\
\hline & Healthy $(n=28)$ & $\begin{array}{l}\text { COPD total } \\
\quad(n=37)\end{array}$ & $\begin{array}{c}\text { COPD } \\
\text { functional } \\
(n=12)\end{array}$ & \\
\hline Amino and keto acids ( $\mu$ mol/l) & & & & \\
\hline tau-methylhistidine & $4.7(0.3)$ & $4.4(0.3)$ & $4.1(0.5)$ & $4.5(0.4)$ \\
\hline Valine & $173.2(7.5)$ & $157.3(6.7)$ & $159.6(10.4)$ & $156.2(8.8)$ \\
\hline Isoleucine & $54.3(2.5)$ & $55.4(2.6)$ & $51.9(4.0)$ & $57.2(3.3)$ \\
\hline Leucine & $95.9(4.0)$ & 87.7 (3.9) & $84.1(4.8)$ & $89.6(5.5)$ \\
\hline BCAAsum & $323.3(13.1)$ & $300.4(12.6)$ & $295.6(18.0)$ & $303.0(16.9)$ \\
\hline Tryptophan & $35.9(1.5)$ & $35.2(1.1)$ & $37.9(1.3)$ & $33.9(1.5)$ \\
\hline Phenylalanine & $43.6(1.48)$ & $44.8(1.6)$ & $43.7(2.0)$ & $45.3(2.2)$ \\
\hline Tyrosine & $48.6(2.4)$ & $48.0(2.0)$ & $50.4(2.4)$ & $49.8(2.8)$ \\
\hline Glycine & $224.1(15.6)$ & $226.8(13.3)$ & $226.8(13.3)$ & $235.7(15.7)$ \\
\hline Glutamate & $49.0(5.4)$ & $40.1(4.2)$ & $43.4(5.7)$ & $38.4(5.7)$ \\
\hline Essential amino acids sum & $746.8(24.4)$ & $724.5(22.7)$ & $716.3(25.2)$ & $728.7(32.2)$ \\
\hline$\alpha$-ketoisocaproic acid (KIC) & $26.5(2.1)$ & $26.2(1.7)$ & $26.1(2.9)$ & $26.3(2.1)$ \\
\hline$\alpha$-ketoisovalerate (KIV) & $14.0(0.7)$ & $13.1(0.7)$ & $12.6(1.1)$ & $13.3(0.9)$ \\
\hline$\alpha$-keto- $\beta$-methylvalerate (KMV) & $14.4(1.3)$ & $15.2(1.0)$ & $14.8(1.7)$ & $15.3(1.3)$ \\
\hline BCKAsum & $45.5(2.9)$ & $54.5(3.2)$ & $53.6(5.4)$ & $55.0(4.1)$ \\
\hline
\end{tabular}




\begin{tabular}{|l|c|c|c|c|}
\hline $\begin{array}{l}\beta \text {-hydroxy } \beta \text {-methylbutyrate } \\
\text { (HMB) }\end{array}$ & $2.2(0.2)$ & $2.1(0.2)$ & $1.8(0.3)$ & $2.2(0.3)$ \\
\hline Clinical markers & $5.5(0.1)$ & $5.8(0.2)$ & $6.1(0.3)^{*}$ & $5.6(0.2)$ \\
\hline Glucose (mmol/L) & $1.2(0.3)$ & $5.7(1.3)^{* *}$ & 6.097 \\
\hline $\begin{array}{l}\text { High sensitive C-Reactive } \\
\text { Protein (mg/L) }\end{array}$ & & & & $5.2(1.3)^{*}$ \\
\hline $\begin{array}{l}\text { Data are mean (SE). Statistics are by unpaired t-test or 1-way ANOVA. BCAAsum = Sum of the branched- } \\
\text { chain amino acids valine, isoleucine and leucine. BCKAsum = Sum of the branched-chain keto acids } \alpha- \\
\text { ketoisocaproic acid, } \alpha \text {-ketoisovalerate and } \alpha \text {-keto- } \beta \text {-methylvalerate. * denotes difference from Healthy, } \\
\text { p<0.05; \# denotes difference from COPD functional group, } p<0.05\end{array}$ \\
\hline
\end{tabular}

Table 4: Whole body production of amino and keto acids

\begin{tabular}{|l|c|c|c|c|}
\hline & Healthy (n=28) & $\begin{array}{c}\text { COPD total } \\
(\mathbf{n = 3 7})\end{array}$ & $\begin{array}{c}\text { COPD } \\
\text { functional } \\
(\mathbf{n}=\mathbf{1 2})\end{array}$ & $\begin{array}{c}\text { COPD } \\
\text { dysfunctional } \\
(\mathbf{n}=\mathbf{2 5})\end{array}$ \\
\hline Glutamate & $172.3(17.7)$ & $221.8(25.3)$ & $263.5(60.9)$ & $203.7(25.0)$ \\
\hline Glutamine & $408.9(29.1)$ & $392.5(24.6)$ & $398.8(41.9)$ & $389.1(31.1)$ \\
\hline Glycine & $191.5(14.7)$ & $187.7(12.7)$ & $185.7(24.9)$ & $188.7(14.8)$ \\
\hline Citrulline & $14.2(0.8)$ & $16.7(0.9)^{*}$ & $16.2(1.3)$ & $\begin{array}{c}17.0(1.2) \\
* p=0.055\end{array}$ \\
\hline Arginine & $81.0(6.0)$ & $102.7(6.4)^{*}$ & $107.1(10.0)^{*}$ & $\begin{array}{c}100.5(8.3) \\
* p=0.065\end{array}$ \\
\hline tau-methylhistidine & $0.77(0.10)$ & $0.86(0.06)$ & $0.86(0.10)$ & $0.87(0.07)$ \\
\hline Leucine & $96.3(4.7)$ & $115.7(5.2)^{*}$ & $113.3(8.8)$ & $116.9(6.5)^{*}$ \\
\hline Phenylalanine & $73.4(5.4)$ & $64.0(3.9)$ & $65.1(6.8)$ & $63.5(4.9)$ \\
\hline
\end{tabular}




\begin{tabular}{|l|c|c|c|c|}
\hline Tyrosine & $56.5(3.7)$ & $52.2(2.8)$ & $54.4(4.8)$ & $51.0(3.6)$ \\
\hline Tryptophan & $10.5(0.8)$ & $9.8(0.5)$ & $10.6(0.9)$ & $9.4(0.6)$ \\
\hline$\alpha$-ketoisocaproic acid (KIC) & $13.3(1.7)$ & $17.2(2.0)$ & $13.8(1.8)$ & $\begin{array}{c}18.6 \\
(2.7)^{*} p=0.093\end{array}$ \\
\hline $\begin{array}{l}\text { Data are in } \mu \mathrm{mol} / \mathrm{kg} \mathrm{ffm} / \mathrm{h}, \text { mean (SE). Statistics are by unpaired t-test or 1-way ANOVA. * denotes } \\
\text { difference from Healthy, p<0.05; \# denotes difference from COPD functional, p<0.05. }\end{array}$ \\
\hline
\end{tabular}







\section{References}


IV pulse of stable tracers

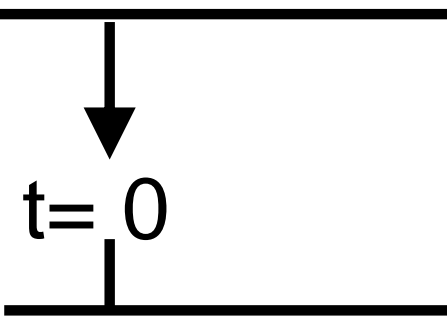

*IV pulse:

Such as L-[ring- ${ }^{13} \mathrm{C}_{6}$ ]-Phenylalanine, L-[ring-D 4 ]Tyrosine, L-[Methyl-

$\mathrm{D}_{3}$ ]Tau-Methylhistidine, L-[Guanido- $\left.{ }^{15} \mathrm{~N}_{2}\right]$-Arginine, L-[4,4,5,5- $\mathrm{D}_{4}-5$ -

$\left.{ }^{13} \mathrm{C}\right]$ Citruline, L- ${ }^{15} \mathrm{~N}_{2}$-Glutamine, L-[1,2-13 $\left.\mathrm{C}_{2}\right]$ Glutamic Acid, $1-{ }^{13} \mathrm{C}$-Glycine,

$\mathrm{L}^{-13} \mathrm{C}_{6}$-Leucine, $\alpha-1-{ }^{13} \mathrm{C}$-ketoisocaproic acid, L-(Indole- $\left.\mathrm{D}_{5}\right)$ Tryptophan

\section{Blood sampling IIII I I | I I}

Cognitive testing

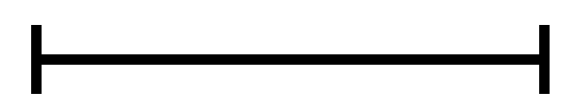

Questionnaires

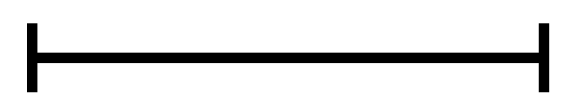

Muscle dynamometry

Figure 1. Overview of study design. Cognitive testing: executive functioning and psychomotor speed. Questionnaires: physical activity, mood status, dietary recall. Muscle dynamometry: upper and lower limb muscle strength. 


\section{A}

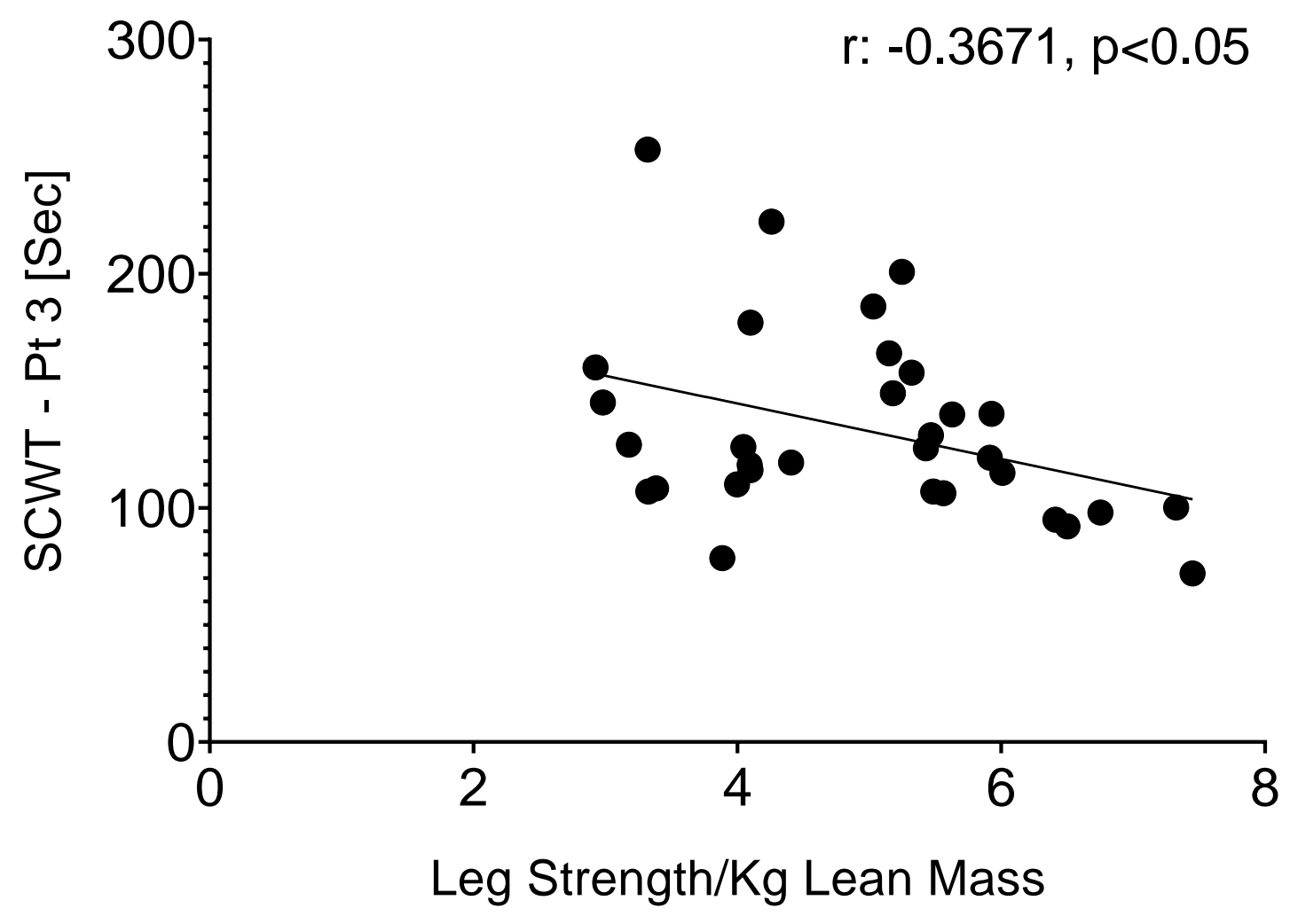

B

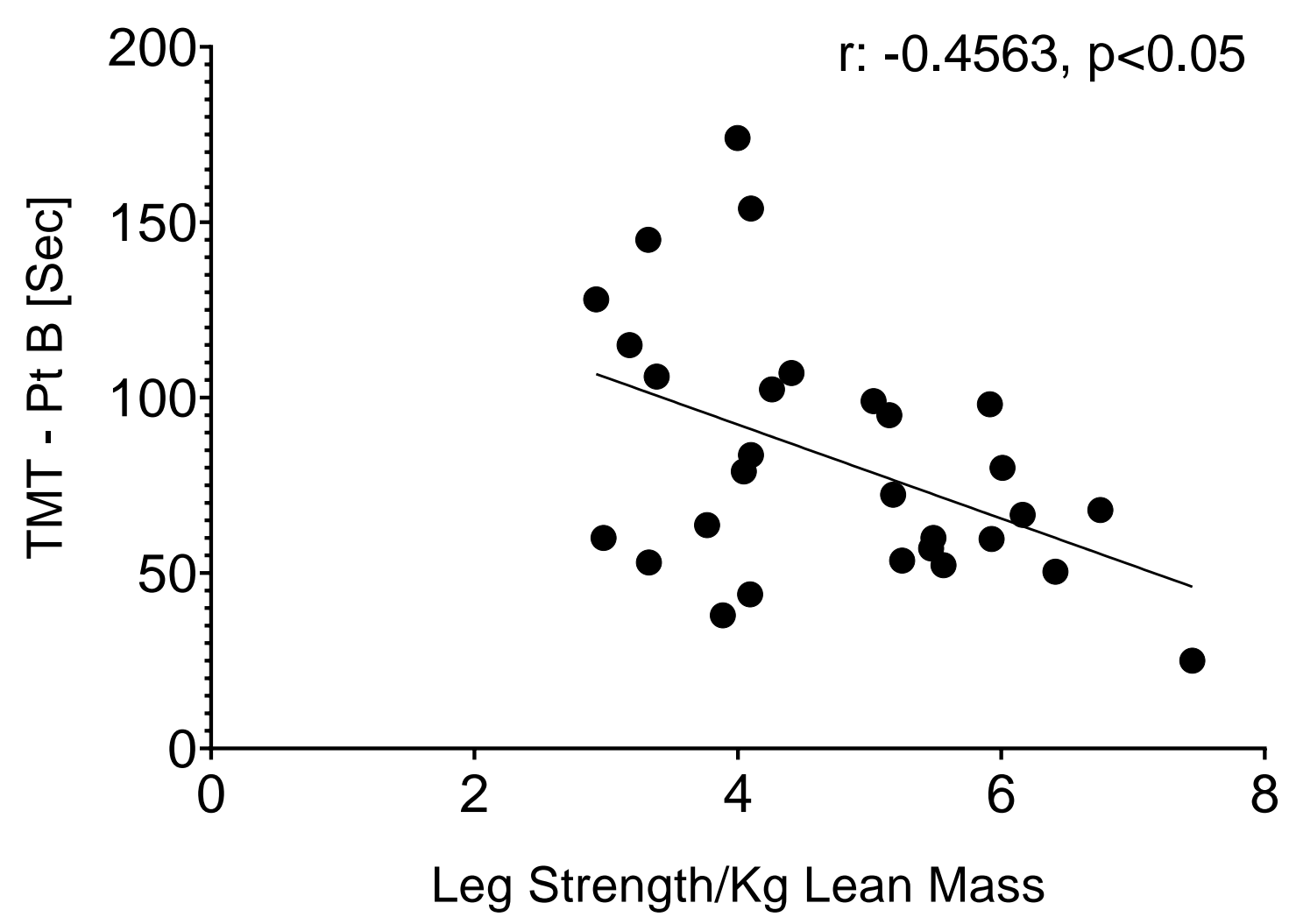

Figure 5. Correlations between muscle function (Leg strength/Kg lean mass) and (A) Stroop Color Word Test performance (SCWT - Pt 3). (B) Trail making test performance (TMT- Pt B) in all COPD patients. Statistics by Pearson correlation. 

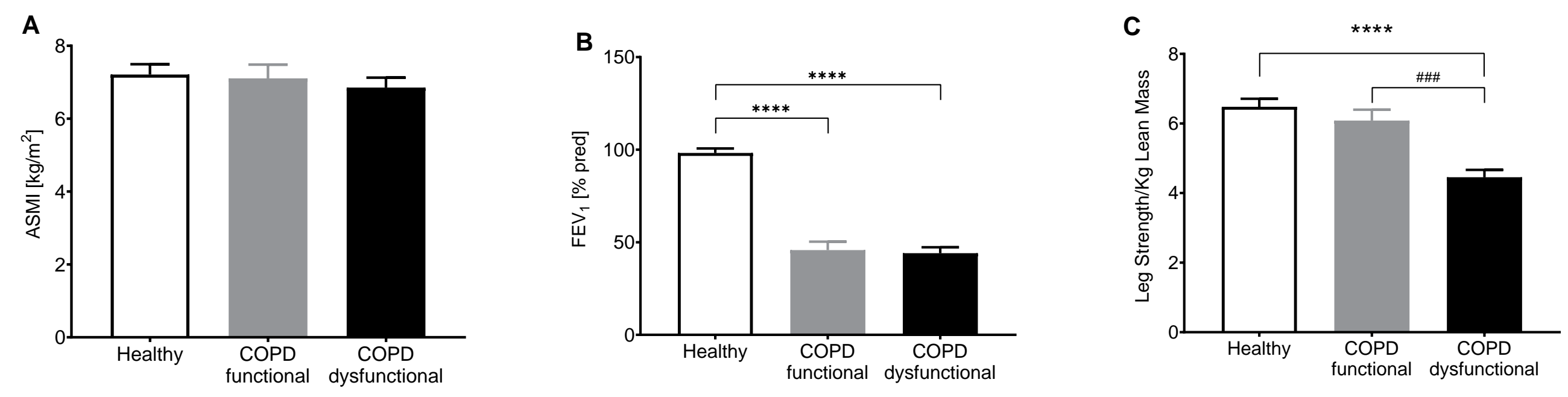

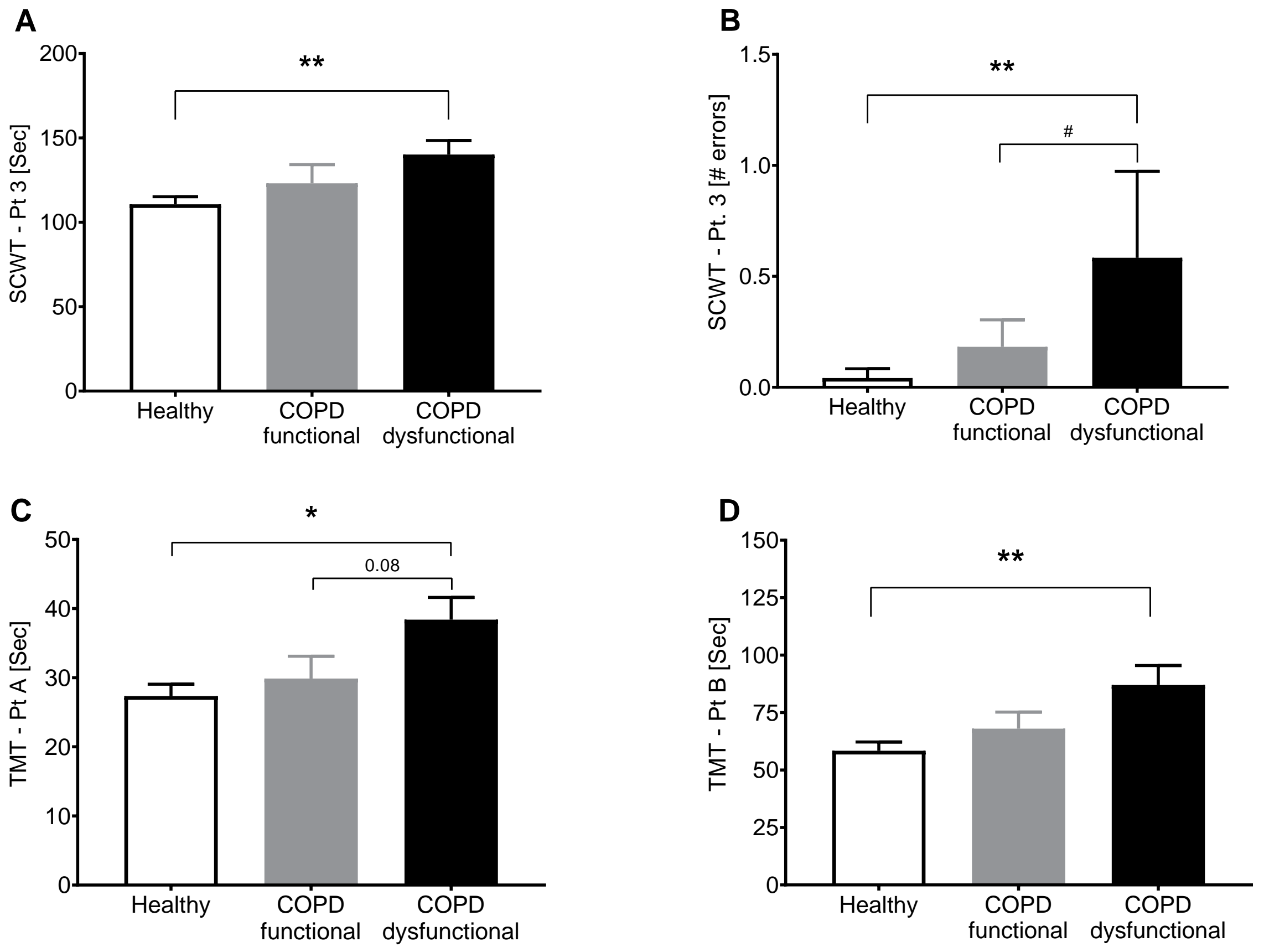